\title{
Two Cases of Primary Ciliary Dyskinesia with Different Responses to Macrolide Treatment
}

\author{
Takashi Kido ${ }^{1}$, Kazuhiro Yatera ${ }^{1}$, Kei Yamasaki ${ }^{1}$, Shuya Nagata ${ }^{1}$, Yasuo Choujin ${ }^{1}$, \\ Chiyo Yamaga ${ }^{1}$, Kanako Hara ${ }^{1}$, Hiroshi Ishimoto ${ }^{1}$, Masanori Hisaoka ${ }^{2}$ and Hiroshi Mukae ${ }^{1}$
}

\begin{abstract}
We herein report two cases of primary ciliary dyskinesia (PCD) with different responses to macrolides. Case 1: a 17-year-old Japanese man with Pseudomonas aeruginosa infection and combined defect of both inner and outer dynein arms in the cilia was unsuccessfully treated with long-term macrolides (clarithromycin, erythromycin, and azithromycin). Case 2: a 70-year-old Japanese man with deficiency of only the inner dynein arm was successfully treated with clarithromycin. Though the reasons for the different responses to macrolides are unclear, differences of ultrastructural abnormalities of the cilia might be one of the predictive factors in PCD just as in Pseudomonas aeruginosa infection.
\end{abstract}

Key words: azithromycin, clarithromycin, dynein arm, immotile cilia, macrolide, primary ciliary dyskinesia

(Intern Med 51: 1093-1098, 2012)

(DOI: 10.2169/internalmedicine.51.6617)

\section{Introduction}

Primary ciliary dyskinesia (PCD), a rare disease estimated to affect 1 in 15,000 to 20,000 individuals, is accompanied by ciliary dysfunction $(1,2)$. The ciliary beat frequency (CBF) is closely associated with specific ultrastructural abnormalities of the cilia in patients with PCD. With regard to the ciliary motility in patients with PCD, Chilvers et al. reported that inner dynein arm (IDA) defect is associated with relatively mild dysfunction, while the presence of a combined defect of both IDA and the outer dynein arm (ODA) is associated with severe dysfunction (3). The ciliary beat is an important primary innate defense mechanism, and ciliary dysfunction leads to upper and lower respiratory diseases such as chronic bronchitis, bronchiectasis, chronic sinusitis and chronic otitis media from infancy in patients with PCD. There are no standardized effective treatment strategies for PCD, although the lung involvement of PCD can develop into severe or fatal respiratory failure. Based on past experiences regarding the effects of treatment with macrolides in patients with diffuse panbronchiolitis (DPB), long-term treatment with macrolides such as clarithromycin (CAM), erythromycin (EM) and azithromycin (AZM) has been empirically applied for the treatment of PCD in Japan (4-9). However, the effects of macrolide therapy in patients with PCD remain controversial. We herein report the cases of two PCD patients with different responses to macrolide treatment.

\section{Case Reports}

Case 1: A 17-year-old Japanese man had been diagnosed and treated for chronic sinusitis, exudative otitis media and bronchial asthma since his childhood. He had experienced repeated hemoptysis, and chest computed tomography (CT) had revealed right middle lobe bronchiectasis and collapse when he was 14 years old. He had been experiencing a productive cough and green sputum for one month prior to admission to our hospital. He was referred and admitted to our hospital via an internal medicine clinic that he initially visited because of hemoptysis, a low-grade fever $\left(37.3^{\circ} \mathrm{C}\right)$ and exertional dyspnea [British Medical Research Council (MRC) dyspnea scale grade 3]. A physical examination upon admission showed blood pressure of $125 / 78 \mathrm{mmHg}$ and a regular pulse of 125 beats/min. Lung auscultation re-

${ }^{1}$ Department of Respiratory Medicine, University of Occupational and Environmental Health, Japan and ${ }^{2}$ Department of Pathology and Oncology, University of Occupational and Environmental Health, Japan

Received for publication September 24, 2011; Accepted for publication January 23, 2012

Correspondence to Dr. Takashi Kido, t-kido@med.uoeh-u.ac.jp 


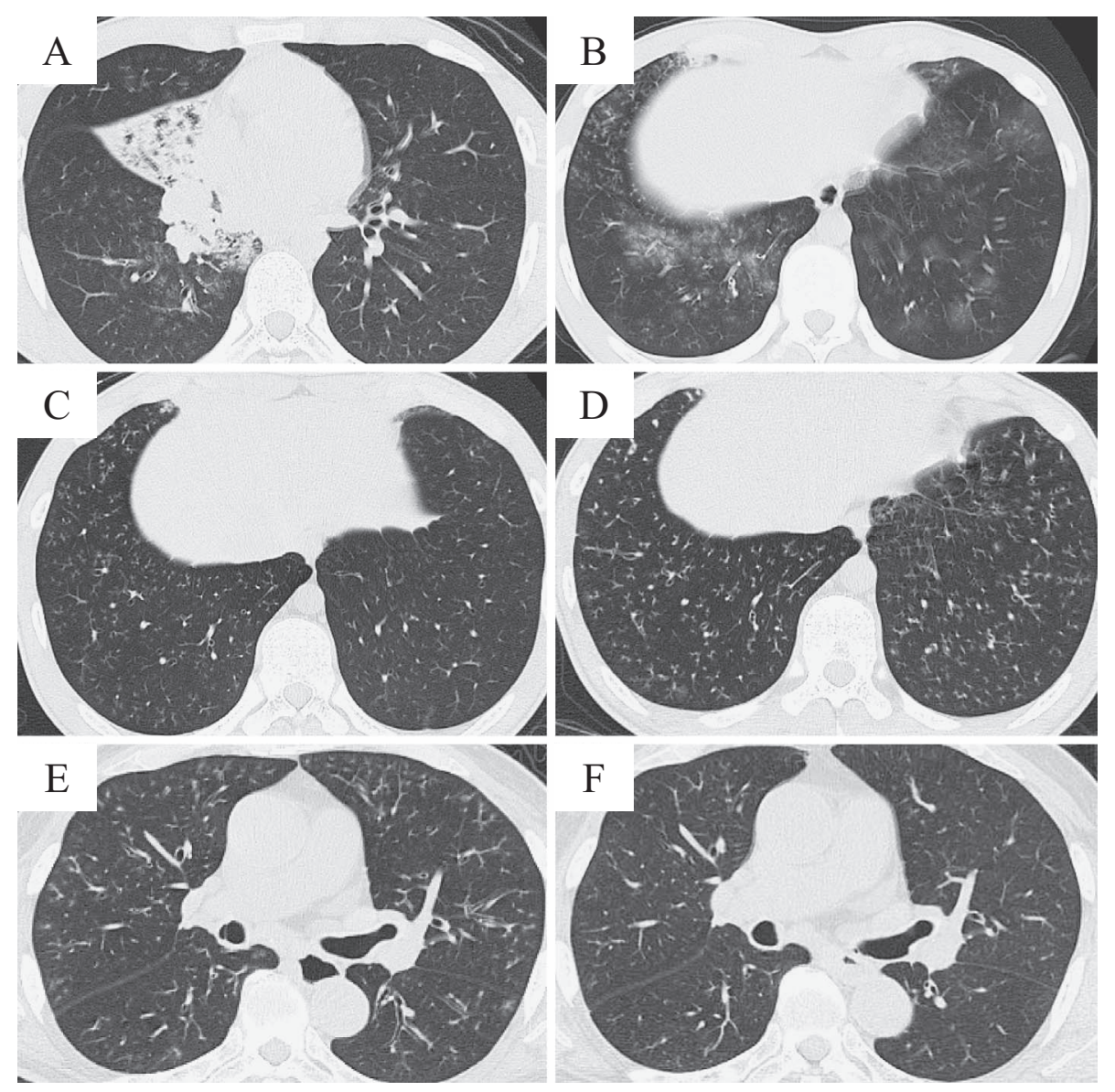

Figure 1. The chest computed tomography (CT) findings of Cases 1 (A to D) and 2 (E, F). The chest CT of Case 1 on the first admission to our hospital showed diffuse fine granular shadows, thickening of the bronchial walls and patchy bilateral ground-glass opacities (A), in addition to the bronchiectasis of the collapsed right middle lobe (B). C: The chest CT findings of Case 1 two months after the first admission, showing improvement of these findings compared to those at the first admission. D: The CT findings of Case 1 sixteen months after the first admission indicating exacerbation of multiple fine granular opacities, even after long-term macrolide treatment with clarithromycin (CAM), erythromycin (EM) and azithromycin (AZM). E: The chest CT findings of Case 2 on admission showed diffuse fine granular shadows and thickening of the bronchial walls. F: The CT findings of Case 2 seven months after the first admission were almost completely improved by the treatment with CAM (200 mg/day).

vealed expiratory wheezes in the right lung. The results of the laboratory data, arterial blood gas analysis and pulmonary function test on admission were as follows: white blood cell count, 15,000/ $\mathrm{L}$; neutrophils, 77.9\%; hemoglobin, $14.9 \mathrm{~g} / \mathrm{dL}$; cold agglutinin, $\times 4 ; \mathrm{pH}, 7.398 ; \mathrm{PaCO}_{2}, 43.6$ Torr; $\mathrm{PaO}_{2}, 83.0$ Torr; VC, $4.99 \mathrm{~L}$; \%VC, 99.0\%; FEV ${ }_{1.0}$, $3.72 \mathrm{~L} / \mathrm{sec} ; \mathrm{FEV}_{1.0}, \% 77.0 \%$. A chest $\mathrm{CT}$ on admission showed diffuse fine granular opacities, bronchial wall thickening and patchy bilateral ground-glass opacities (Fig. 1A) in addition to right middle lobe collapse and bronchiectasis (Fig. 1B).

The patient was diagnosed to have pneumonia in the right middle lobe and hemoptysis from the same lesion. He started to receive antimicrobials (meropenem) on the day of admission, and the chest CT findings 2 months after the first admission were improved (Fig. 1C). Pseudomonas aeruginosa was detected continuously in cultured sputum, and re- petitive acute exacerbations of lower respiratory tract infections occurred in spite of long-term treatment with CAM (200 mg/day) (Fig. 2). Flexible bronchoscopy at 3 months after the first admission revealed a massive amount of yellowish bronchial secretions from the right middle lobe bronchus (Fig. 3A). An electron microscopic examination of biopsy specimens obtained from the bronchial mucosa revealed the combined defect of both IDA and ODA in the cilia (Fig. 3B). Based on these findings, the patient was diagnosed to have PCD. CAM was changed to EM (400 mg/ day) beginning 3 months after the first admission, but the symptoms of the lower respiratory tract infections and hemoptysis were still uncontrolled.

Because the right middle lobe was considered to be the major source of infection and hemoptysis, surgical resection of the right middle lobe was performed 4 months after the first admission. After switching macrolides from CAM to 


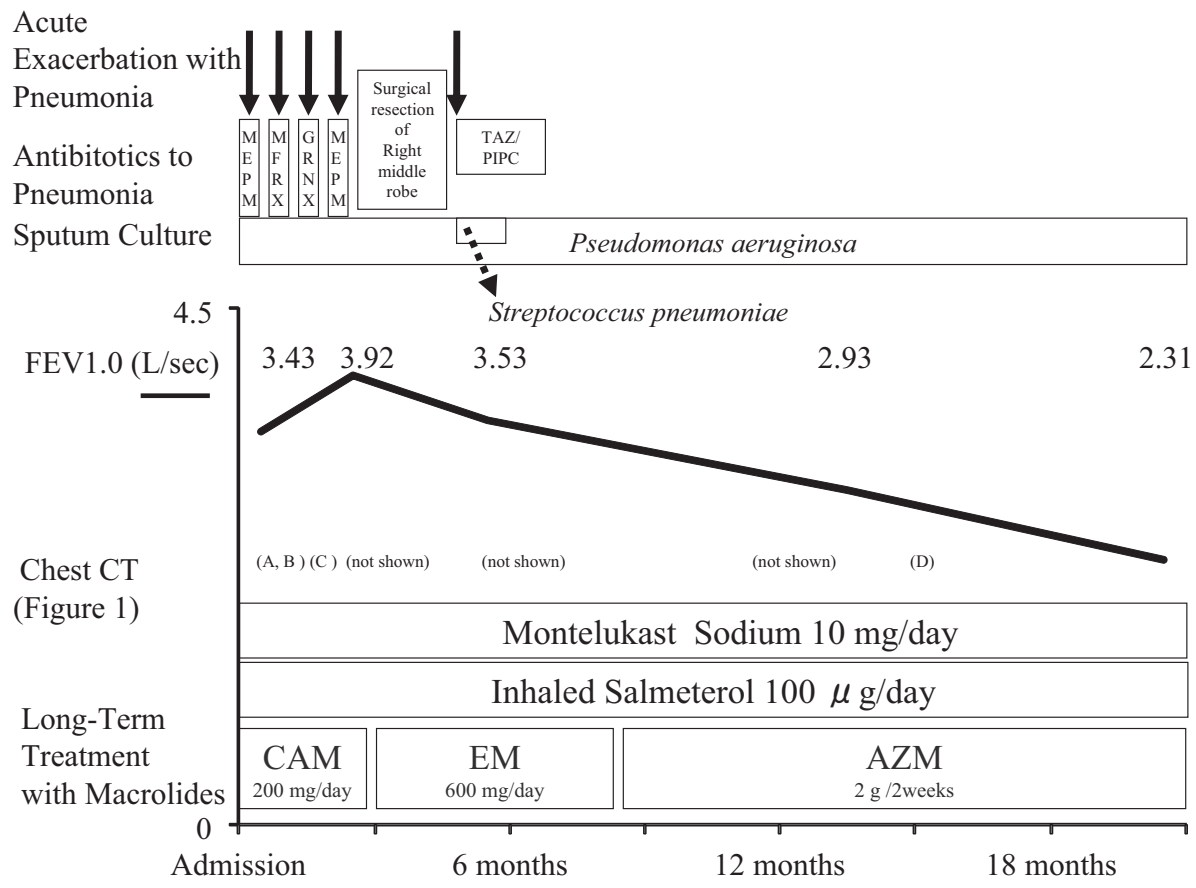

Figure 2. The clinical course of Case 1. In spite of the treatment with three different types of macrolides (CAM, EM and AZM) and the surgical resection of the right middle lobe, the findings of the chest CT scan and pulmonary function tests were worsened. However, after starting the treatment with azithromycin, the frequency of acute exacerbations of the lower respiratory tract infections (black arrows) was decreased. GRNX: garenoxacin, MEPM: meropenem, MFRX: moxifloxacin, TAZ/PIPC: tazobactam/piperacillin

EM and the right middle lobe resection, the frequency of acute exacerbations of lower respiratory tract infections was decreased, although diffuse fine granular shadows and bronchial wall thickening on chest CT at 6 months after the first admission were exacerbated. EM was changed to AZM (2 g/ 2 weeks) beginning 8 months after the first admission, and no acute exacerbations were observed after the initiation of AZM. However, the findings of chest CT scans were worsened at 12 months and 16 months after the first admission (Fig. 1D), and a deterioration of the $\mathrm{FEV}_{1.0}$ was observed (Fig. 2). He also had a productive cough continuously after the initiation of the treatment.

Case 2: A 70-year-old Japanese man with a medical history of chronic sinusitis was admitted to our hospital after developing a persistent cough and yellow sputum. His chest CT showed diffuse fine granular shadows and thickened bronchial walls (Fig. 1E). Sputum microbiology revealed Haemophilus influenzae and Moraxella catarrhalis. The bronchoalveolar lavage fluid obtained from the right $\mathrm{B}^{5}$ contained an increased total cell count $\left(13.7 \times 10^{5} / \mathrm{mL}\right)$ with a high fraction of neutrophils $(86.7 \%)$, a low fraction of lymphocytes $(3.53 \%)$ and a low $\mathrm{CD} 4 / 8$ ratio $(0.3)$. The deficiency of IDA in the cilia was revealed by the electron microscopic examination of the bronchial mucosal biopsy specimens (Fig. 3C), suggesting a diagnosis of PCD. Longterm treatment with CAM (200 mg/day) was started after the diagnosis and was maintained for 11 months. This treatment improved his symptoms of productive cough and puru- lent sputum. No acute exacerbations were observed after the initiation of CAM. The chest CT findings were also gradually improved at one month (showing slightly fine granular shadows) and 7 months (Fig. 1F, almost completely improved) after the initiation.

\section{Discussion}

We herein report two cases of PCD treated with long-term macrolides that showed different treatment responses. Case 1 had a combined defect of both IDA and ODA in the cilia and also had Pseudomonas aeruginosa infection. The patient showed a poor response to three different types of macrolides on both radiological findings and pulmonary function tests, although the frequency of acute exacerbations of lower respiratory tract infections was improved with AZM. On the other hand, Case 2 had only an IDA defect in the cilia, and demonstrated a good response to CAM.

PCD is a genetic disease associated with abnormal ciliary structure and function. The ciliary beat is an important primary innate defense mechanism and the dysfunction impairs the mucociliary clearance which protects the lungs from pathogens, pollutants and allergens. Repeated lower respiratory tract infections result in the progressive destruction of the lung that can lead to severe and fatal pulmonary dysfunction $(10,11)$. Thus, the severity of PCD, the degree of ciliary dysfunction and the specific ultrastructural abnormalities of the cilia are closely associated with each other $(2,3)$. 


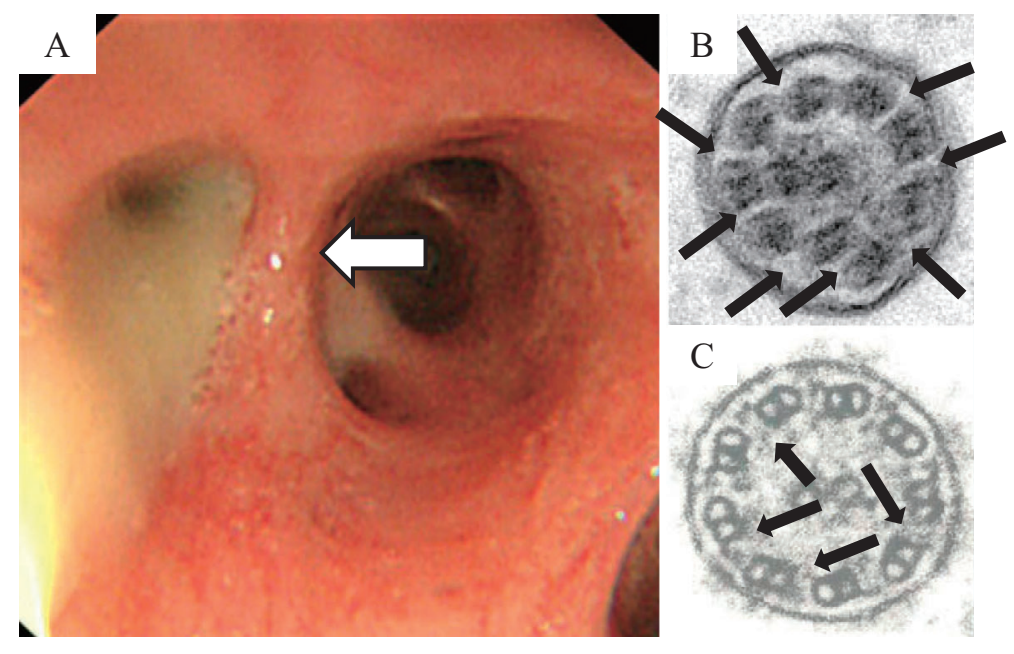

Figure 3. The flexible bronchoscopic and electron microscopic findings of Cases 1 (A, B) and 2 (C). A: The flexible bronchoscopic findings of Case 1 three months after the first admission, revealing a massive amount of yellowish bronchial secretions from the right middle lobe bronchi (white arrow). B: Combined defect of both the inner dynein arm (IDA) and the outer dynein arm (ODA) in the cilia (black arrows) was detected by the electron microscopic examination of the biopsy specimens from the bronchial mucosa obtained from Case 1. C: The defect of the IDA (black arrows) in the cilia was revealed by electron microscopy in the biopsy specimens of the bronchial mucosa obtained from Case 2.

Table 1. Summarized Case Reports of Primary Ciliary Dyskinesia (PCD) in Patients with Detected Ultrastructural Abnormalities in the Cilia who Were Treated with Long-term Macrolides (Homma et al and Ishiguro et al. Reported 8 and 4 Cases of PCD, Respectively, But Only One and Two Cases Included the Details of the Ultrastructural Abnormalities in the Cilia, Respectively).

\begin{tabular}{|c|c|c|c|c|c|}
\hline \multirow{2}{*}{$\begin{array}{l}\text { Author / Year } \\
\text { (Reference } \\
\text { number). }\end{array}$} & \multirow{2}{*}{$\begin{array}{l}\text { Type of } \\
\text { Ultrastructual } \\
\text { Abnormality in Cilia }\end{array}$} & \multirow{2}{*}{$\begin{array}{l}\text { Bacterological findings } \\
\text { of sputum culture }\end{array}$} & \multicolumn{3}{|c|}{ Response to macrolide treatment } \\
\hline & & & $\begin{array}{l}\text { Types of } \\
\text { macrolides }\end{array}$ & $\begin{array}{l}\text { Clinical } \\
\text { response }\end{array}$ & $\begin{array}{l}\text { Radiological } \\
\text { response }\end{array}$ \\
\hline $\begin{array}{l}\text { Homma, et } \\
\text { al. } 1999 \text { (4). }\end{array}$ & IDA and ODA & Pseudomonus aeruginosa & EM & $\begin{array}{l}\text { Not } \\
\text { improved }\end{array}$ & $\begin{array}{l}\text { Not } \\
\text { improved }\end{array}$ \\
\hline $\begin{array}{l}\text { Itoh, et al. } \\
2002(5)\end{array}$ & IDA & Pseudomonus aeruginosa & $\begin{array}{l}\text { CAM, } \\
\text { AZM }\end{array}$ & $\begin{array}{l}\text { Improved } \\
\text { with AZM }\end{array}$ & $\begin{array}{l}\text { Improved } \\
\text { with AZM }\end{array}$ \\
\hline $\begin{array}{l}\text { Ishiga, et al. } \\
2005(6)\end{array}$ & IDA and ODA & $\begin{array}{l}\text { Klebsiella pneumoniae } \\
\text { Pseudomonus aeruginosa }\end{array}$ & CAM & $\begin{array}{l}\text { Not } \\
\text { improved }\end{array}$ & $\begin{array}{l}\text { Not } \\
\text { improved }\end{array}$ \\
\hline \multirow[t]{2}{*}{$\begin{array}{l}\text { Ishiguro, et } \\
\text { al. } 2009 \text { (7). }\end{array}$} & IDA and ODA & $\begin{array}{l}\text { Streptococcus pneumoniae } \\
\text { Haemophilus influenzae }\end{array}$ & CAM & Improved & NA \\
\hline & IDA and ODA & $\begin{array}{l}\text { Klebsiella pneumoniae } \\
\text { Aspergillus fumigatus } \\
\text { Proteus mirabilis }\end{array}$ & CAM & Improved & NA \\
\hline $\begin{array}{l}\text { Yoshioka,et } \\
\text { al. } 2010(8) .\end{array}$ & IDA & Staphylococcus aureus & CAM & Improved & Improved \\
\hline $\begin{array}{l}\text { Tada, et al. } \\
2011(9)\end{array}$ & IDA & Haemophilus influenzae & CAM & Improved & Improved \\
\hline \multirow{2}{*}{$\begin{array}{l}\text { Present } \\
\text { cases. } \\
2012 \text {. }\end{array}$} & IDA and ODA & Pseudomonus aeruginosa & $\begin{array}{l}\text { CAM, EM, } \\
\text { AZM }\end{array}$ & $\begin{array}{l}\text { Improved } \\
\text { with AZM }\end{array}$ & $\begin{array}{l}\text { Not } \\
\text { improved }\end{array}$ \\
\hline & IDA & Haemophilus influenzae & CAM & Improved & Improved \\
\hline
\end{tabular}

The four cases with defect of IDA in the cilia were all improved by long-term treatment with macrolides in terms of both the clinical symptoms (such as cough, sputum production and frequency of acute exacerbations) and radiological findings. On the other hand, two of five patients with a combined defect of both IDA and ODA in the cilia did not respond to the treatment with macrolides regarding clinical respiratory symptoms, and three of five patients did not respond regarding the radiological findings.

Chilvers et al. reported that the mean CBF was $12.8 /$ second in normal subjects, whereas the mean $\mathrm{CBF}$ was 8.1, 2.3 and
$0.8 /$ second in patients with IDA, ODA, and dual IDA and ODA defect of the cilia, respectively (3). The ideal agents to 
treat patients with PCD should overcome the ciliary dysfunction (10). However, there are currently no effective therapeutic strategies that correct this inborn error, and macrolides do not seem to have an effect on the CBF (12). Beta2-adrenoreceptor agonists have been shown to enhance $\mathrm{CBF}$ in vitro $(13,14)$, though there is little data indicating that such agents improve the function of dyskinetic cilia. We also used this agent in Case 1, resulting in no obvious clinical effects. L-arginine is thought to have a potential role of enhancing $\mathrm{CBF}$ via increasing the airway nitric oxide levels, but the infusion of L-arginine to patients with PCD does not improve their pulmonary function and does not increase nasal nitric oxide levels high enough to reach the same levels of healthy individuals (15).

Accordingly, it is difficult to cure the patients with PCD completely, and the management of lower respiratory tract infections in these patients is very important to delay or stop the disease progression $(10,11)$. The respiratory management of PCD is basically based on that for cystic fibrosis (CF) in European and North American countries, and is based on that of DPB in Japan. Many therapeutic agents have been tried for PCD, however, there have been no randomized controlled trials, and there are currently no standardized therapeutic strategies $(10,11)$. From the clinical experiences of the efficacy of macrolides in patients with DPB, long-term treatment with macrolides has been used for patients with PCD in Japan (4-9). The nine reported cases of PCD, including the present two cases, with ultrastructural abnormalities in the cilia treated by long-term macrolide administration are summarized in Table 2. Four patients with a defect of only IDA in the cilia were all clinically and radiologically improved by long-term treatment with macrolides. On the other hand, two of five patients with a combined defect of both IDA and ODA in the cilia did not respond to the treatment with macrolides in clinical respiratory symptoms, and also three of five patients did not respond based on the radiological findings. From these findings, we speculate that the different severity of ultrastructural abnormalities of the cilia may be one of the predictive factors of response to macrolide treatment in patients with PCD. While PCD is a rare disease and it is not easy to perform a protective study, further accumulation of evidence is expected to confirm our speculation. Persistent lower respiratory tract Pseudomonas aeruginosa infection is also well known as one of the poor prognosis factors of lung diseases including DPB and PCD $(2,16,17)$. As listed in Table 2, Pseudomonas aeruginosa was detected from sputum culture in all three cases without a response to long-term treatment with macrolides radiologically, supporting the results of these previous reports $(2,16,17)$. Considering that Pseudomonas aeruginosa was detected in three of five cases with a combined defect of both IDA and ODA in the cilia, and one of four cases with a defect of IDA in the cilia (Table 2), the different severity of ultrastructural abnormalities of the cilia may also be closely associated with Pseudomonas aeruginosa infection. Other than these factors, disease severity and genetic heterogeneity have been considered to be predictive factors of the response to macrolides, and they also seem to be closely related to ultrastructural abnormalities of the cilia $(1,8,18,19)$.

Long-term treatment with macrolides has been shown to improve the prognosis of DPB (20). The 14- and 15member-ring macrolides improved the clinical outcomes of patients with DPB, and there has recently been much attention focused on the use of macrolides as a potential therapy for other inflammatory airway disorders, such as bronchial asthma, bronchiectasis, chronic obstructive pulmonary disease, chronic rhinosinusitis, cystic fibrosis and obliterative bronchiolitis (21). As antimicrobial agents, macrolide antibiotics including CAM are widely used as first-line agents to treat acute bacterial infections such as community-acquired pneumonia (22). In addition to their antimicrobial activities, macrolides inhibit airway mucus secretion, decrease the infiltration and prolongation of the activation of inflammatory cells such as lymphocytes, macrophages and neutrophils in the lungs, and decrease the production of cytokines such as interleukin (IL) -1, -2, -6 and -8, and tumor necrosis factor$\alpha$ from the lungs (23-30). The mechanisms of action of the macrolides are thought to be due to the immunomodulatory effects of the agents, rather than their direct antimicrobial activities (21). Based on these reports, we speculated that macrolides could improve the airway inflammation in patients with PCD through their immunomodulatory activities. The differences in the effects of each macrolide on the immune system are still unclear; AZM seemed to be more effective than the other macrolides in Case 1 having a combined defect of both IDA and ODA with persistent lower respiratory tract infection of Pseudomonas aeruginosa, reducing the frequency of acute exacerbations. It suggests that to stop the disease progression of PCD completely is difficult, but using AZM in an earlier stage of PCD may be better to delay the disease progression in severe cases.

In conclusion, this report describes two cases of PCD with different responses to macrolide treatment. Though the reason for the differing responses to macrolides is still unclear, differences in ultrastructural abnormalities of the cilia might be one of the predictive factors of the response to macrolides in PCD. While PCD is a rare disease, further accumulation of the evidence regarding the relationship between the response to macrolides and ultrastructural cilial abnormalities is expected to confirm our speculation. Macrolides may have the potential to ameliorate the natural course of PCD with mild ciliary dysfunction via the modification of the activities of the immune system, although the complete suppression of disease progression is difficult to be accomplished, especially in severe cases. AZM seems to be a better choice than other macrolides for patients with PCD in order to reduce the frequency of acute exacerbations and delay the disease progression, according to the clinical course of Case 1.

The authors state that they have no Conflict of Interest (COI). 


\section{References}

1. Hogg C. Primary ciliary dyskinesia: when to suspect the diagnosis and how to confirm it. Paediatr Respir Rev 10: 44-50, 2009.

2. Noone PG, Leigh MW, Sannuti A, et al. Primary ciliary dyskinesia: diagnostic and phenotypic features. Am J Respir Crit Care Med 169: 459-467, 2004.

3. Chilvers MA, Rutman A, O'Callaghan C. Ciliary beat pattern is associated with specific ultrastructural defects in primary ciliary dyskinesia. J Allergy Clin Immunol 112: 518-524, 2003.

4. Homma S, Kawabata M, Kishi K, et al. Bronchiolitis in Kartagener's syndrome. Eur Respir J 14: 1332-1339, 1999.

5. Itoh M, Kishi $\mathrm{K}$, Nakamura $\mathrm{H}$, et al. A case of immotiledyskinetic cilia syndrome responding to clenbuterol hydrochloride and azithromycin. Nihon Kokyuki Gakkai Zasshi 40: 617-621, 2002 (in Japanese, Abstract in English).

6. Ishiga T, Tanigawa M, Ichioka M, Saito K. A case of Kartagener's syndrome. Nihon Kokyuki Gakkai Zasshi 43: 155-159, 2005 (in Japanese, Abstract in English).

7. Ishiguro T, Takayanagi N, Hijikata N, et al. Primary ciliary dyskinesia: A case report and comparison with 4 previous cases. Nihon Kokyuki Gakkai Zasshi 47: 242-248, 2009 (in Japanese, Abstract in English).

8. Yoshioka D, Sakamoto N, Ishimatsu Y, et al. Primary ciliary dyskinesia that responded to long-term, low-dose clarithromycin. Intern Med 49: 1437-1440, 2010.

9. Tada M, Takayanagi N, Ishiguro T, et al. Primary ciliary dyskinesia improved by clarithromycin. J Jpn Soc Rsep Endocscopy 33: 342-347, 2011 (in Japanese, Abstract in English).

10. Amirav I, Cohen-Cymberknoh M, Shoseyov D, Kerem E. Primary ciliary dyskinesia: prospects for new therapies, building on the experience in cystic fibrosis. Paediatr Respir Rev 10: 58-62, 2009.

11. Fauroux B, Tamalet A, Clement A. Management of primary ciliary dyskinesia: the lower airways. Paediatr Respir Rev 10: 55-57, 2009.

12. Takeyama K, Tamaoki J, Chiyotani A, Tagaya E, Konno K. Effect of macrolide antibiotics on ciliary motility in rabbit airway epithelium in-vitro. J Pharm Pharmacol 45: 756-758, 1993.

13. Kanthakumar K, Taylor G, Tsang KW, et al. Mechanisms of action of Pseudomonas aeruginosa pyocyanin on human ciliary beat in vitro. Infect Immun 61: 2848-2853, 1993.

14. Kanthakumar K, Cundell DR, Johnson M, et al. Effect of salmeterol on human nasal epithelial cell ciliary beating: inhibition of the ciliotoxin, pyocyanin. Br J Pharmacol 112: 493-498, 1994.

15. Grasemann H, Gartig SS, Wiesemann HG, Teschler H, Konietzko $\mathrm{N}$, Ratjen F. Effect of L-arginine infusion on airway NO in cystic fibrosis and primary ciliary dyskinesia syndrome. Eur Respir J 13: 114-118, 1999.

16. Poletti V, Casoni G, Chilosi M, Zompatori M. Diffuse panbronchiolitis. Eur Respir J 28: 862-871, 2006.

17. Kennedy MP, Noone PG, Leigh MW, et al. High-resolution CT of patients with primary ciliary dyskinesia. AJR Am J Roentgenol 188: 1232-1238, 2007.

18. Blouin JL, Meeks M, Radhakrishna U, et al. Primary ciliary dyskinesia: a genome-wide linkage analysis reveals extensive locus heterogeneity. Eur J Hum Genet 8: 109-118, 2000.

19. Leigh MW, Pittman JE, Carson JL, et al. Clinical and genetic aspects of primary ciliary dyskinesia/Kartagener syndrome. Genet Med 11: 473-487, 2009.

20. Kudoh S, Uetake T, Hagiwara $K$, et al. Clinical effects of lowdose long-term erythromycin chemotherapy on diffuse panbronchiolitis. Nihon Kyobu Shikkan Gakkai Zasshi 25: 632-642, 1987 (in Japanese, Abstract in English).

21. Crosbie PA, Woodhead MA. Long-term macrolide therapy in chronic inflammatory airway diseases. Eur Respir J 33: 171-181, 2009.

22. Mandell LA, Wunderink RG, Anzueto A, et al. Infectious Diseases Society of America/American Thoracic Society consensus guidelines on the management of community-acquired pneumonia in adults. Clin Infect Dis 44 (Suppl 2): S27-S72, 2007.

23. Konno S, Adachi M, Asano $\mathrm{K}$, et al. Influences of roxithromycin on cell-mediated immune responses. Life Sci 51: PL107-PL112, 1992.

24. Kadota J, Sakito O, Kohno S, et al. A mechanism of erythromycin treatment in patients with diffuse panbronchiolitis. Am Rev Respir Dis 147: 153-159, 1993.

25. Oishi K, Sonoda F, Kobayashi S, et al. Role of interleukin-8 (IL8 ) and an inhibitory effect of erythromycin on IL-8 release in the airways of patients with chronic airway diseases. Infect Immun 62: 4145-4152, 1994.

26. Takizawa H, Desaki M, Ohtoshi T, et al. Erythromycin suppresses interleukin 6 expression by human bronchial epithelial cells: a potential mechanism of its anti-inflammatory action. Biochem Biophys Res Commun 210: 781-786, 1995.

27. Hayakawa H, Sato A, Imokawa S, et al. Diffuse panbronchiolitis and rheumatoid arthritis-associated bronchiolar disease: similarities and differences. Intern Med 37: 504-508, 1998.

28. Iiboshi $\mathrm{H}$, Ashitani $\mathrm{J}$, Katoh $\mathrm{S}$, et al. Long-term treatment with theophylline reduces neutrophils, interleukin- 8 and tumor necrosis factor-alpha in the sputum of patients with chronic obstructive pulmonary disease. Pulm Pharmacol Ther 20: 46-51, 2007.

29. Ishimoto $H$, Mukae $H$, Sakamoto $N$, et al. Different effects of telithromycin on MUC5AC production induced by human neutrophil peptide-1 or lipopolysaccharide in NCI-H292 cells compared with azithromycin and clarithromycin. J Antimicrob Chemother 63: 109-114, 2009.

30. Morinaga $\mathrm{Y}$, Yanagihara K, Miyashita $\mathrm{N}$, et al. Azithromycin, clarithromycin and telithromycin inhibit MUC5AC induction by Chlamydophila pneumoniae in airway epithelial cells. Pulm Pharmacol Ther 22: 580-586, 2009.

(C) 2012 The Japanese Society of Internal Medicine http://www.naika.or.jp/imindex.html 